\title{
Does Subclinical Hypothyroidism Affect Hospitalization Outcomes and Mortality in Congestive Cardiac Failure Patients?
}

\author{
Shanan Mahal ${ }^{1}$, Sorabh Datta ${ }^{1}$, Virendrasinh Ravat ${ }^{2}$, Priya Patel ${ }^{3}$, Bipin Saroha ${ }^{4}$, Rikinkumar S. Patel ${ }^{5}$ \\ 1. Department of Internal Medicine, Providence Hospital, Washington DC, USA 2. Department of Infectious Disease, \\ Clinical Infectious Disease Specialist, Las Vegas, USA 3. Department of Psychiatry, Windsor University School of \\ Medicine, Philadelphia, USA 4. Internal Medicine, University of Chicago Medical Center, Chicago, USA 5. Department \\ of Psychiatry, Griffin Memorial Hospital (odmhsas), Norman, USA
}

Corresponding author: Rikinkumar S. Patel, rpatel_09@arcadia.edu

\section{Abstract \\ Objective}

This study aimed to determine the differences in hospitalization outcomes among patients admitted for congestive cardiac failure (CCF) with underlying subclinical hypothyroidism (SCH).

\section{Methods}

This retrospective case-control study used data from the nationwide inpatient sample (NIS) for the years 2012-2014. We identified cases with CCF as the primary diagnosis and SCH as the secondary diagnosis using validated ICD-9-CM codes and controls with CCF only. The differences in hospitalization outcomes and hospital characteristics were quantified using the multinomial logistic regression model (adjusted odds ratio (aOR)).

\section{Results}

A total of 143,735 CCF patients were enrolled in this study, and 73,440 cases had IH. About $31.8 \%$ of SCH patients were hospitalized for more than four days (median) compared to $44.7 \%$ patients without $\mathrm{SCH}$ ( $\mathrm{P}<$ .001). The median hospitalization charges per admission for CCF was $\$ 20,312$. CCF patients with SCH had lower odds of longer hospitalization $(\mathrm{aOR}=.709,95 \% \mathrm{CI} .660-.762, \mathrm{P}<.001)$ and higher hospitalization charges $(\mathrm{aOR}=.783,95 \%$ CI .728-.841, $\mathrm{P}<.001)$ compared to CCF patients without SCH. CCF patients with SCH had two times higher odds of minor morbidity $(\mathrm{aOR}=2.276 ; 95 \% \mathrm{CI} 2.105-2.462 ; \mathrm{P}<.001)$ but lower odds of major morbidity (aOR $=.783$; 95\% CI .728-.841; P $<.001$ ). Inpatient mortality with SCH patients $(2 \%)$ compared to $3.6 \%$ patients without SCH $(\mathrm{P}<.001)$. CCF patients with SCH had lower odds of in-hospital mortality ( $\mathrm{aOR}=.547 ; 95 \% \mathrm{CI} .496-.604 ; \mathrm{P}<.001)$. CCF patients with SCH had higher odds of being seen in rural non-teaching hospitals ( $\mathrm{aOR}=1.696 ; 95 \%$ CI 1.572-1.831; $\mathrm{P}<.001)$. Also, CCF patients with SCH had the highest likelihood of presence in the western region of the United States ( $\mathrm{OOR}=149.924 ; 95 \%$ CI $110.497-$

Received 06/01/2018

Review began 06/05/2018 Review ended 06/06/2018 Published 06/08/2018

\section{() Copyright 2018}

Mahal et al. This is an open access article distributed under the terms of the Creative Commons Attribution License CC-BY 3.0., which permits unrestricted use, distribution, and reproduction in any medium, provided the original author and source are credited.
203.419; $\mathrm{P}<.001$ ) followed by the southern region ( $\mathrm{aOR}=31.431 ; 95 \% \mathrm{CI} 26.066-37.900 ; \mathrm{P}<.001)$.

\section{Conclusions}

Among CCF with SCH patients during hospitalization, we observed a variation in hospitalization outcomes, including inpatient length of stay and cost, morbidity, and in-hospital mortality. We found no significant increase in mortality and major morbidity in CCF patients with SCH. There were differences in the hospital characteristics between CCF patients with and without SCH. Thus, hospital bed size, location, and teaching status act as predictors for a co-diagnosis of SCH in CCF. Further research is needed to guide the development of clinical care models for targeting early diagnosis and treatment to determine whether thyroid hormone replacement would be beneficial for CCF patients with SCH and improve quality of care in these patients.

Categories: Cardiology, Endocrinology/Diabetes/Metabolism, Quality Improvement

Keywords: hospitalization, ccf, heart failure, outcomes, iatrogenic hypothyroidism, subclinical hypothyroidism

\section{Introduction}

Subclinical hypothyroidism (SCH) is a condition in which an elevated serum thyrotropin level (TSH) is present in combination with the normal range of serum free T4 level. This condition occurs in $3 \%$ to $8 \%$ of the general population. The occurrence of subclinical hypothyroidism varies among people, with a higher incidence associated with increasing age, female sex, and iodine deficiency [1-2]. There is little evidence of the clinical importance and therapy (levothyroxine) for treating subclinical hypothyroidism with a mild elevation of serum TSH ( $<10 \mathrm{mIU} / \mathrm{L})$. Treatment is generally recommended for persons 70 years of age or younger who have thyrotropin levels of at least 10 mIU per liter, although long-term benefits are still 
Subclinical hypothyroidism falls under the category of primary hypothyroidism, which has an etiology that is very similar to overt hypothyroidism [3]. Most cases of SCH are caused by chronic lymphocytic thyroiditis (goitrous Hashimoto's thyroiditis and atrophic thyroiditis). According to previous studies, an autoimmune disorder of the thyroid gland decreased thyroid hormone production in patients with acquired mild, subclinical hypothyroidism [3]. Other causes of primary hypothyroidism could be iatrogenic therapies like radioactive iodine treatment or external radiation therapy that destroy thyroid tissue [3].

Thyroid hormones have a wide range of effects on the human body, among which the cardiovascular system is one of the primary target organs of the thyroid hormone. The most prominent effect of hypothyroidism on patients is increased systemic vascular resistance and impaired systolic and diastolic cardiac function. This same study found that a slower rate of left ventricular relaxation might critically reduce ventricular filling during exercise and result in left ventricular systolic dysfunction [4]. Subclinical hypothyroidism is associated with an increased incidence of congestive heart failure (CHF) at TSH levels $>10 \mathrm{mIU} / \mathrm{L}$. However, the effects of SCH in patients with pre-existing CHF have not been studied (Kannan L, Morley M, Brandimarto J, Cappola TP, and Cappola AR. Thyroid dysfunction in heart failure is associated with cardiovascular outcomes: the Penn Heart Failure Study. ENDO 2017. April 2, 2017). An older population with $\mathrm{SCH}$ and a high CV risk appears to be at an increased risk of incident heart failure and $\mathrm{SCH}$. A recent cardiac magnetic resonance spectroscopic study demonstrated that early cardiac bio-energetic impairments in $\mathrm{SCH}$ patients are reversible with levothyroxine therapy [5].

In previous meta-analysis studies, the association between subclinical hypothyroidism and the risk of mortality did not differ among patients with and without underlying cardiac failure, whereas in a subsequent meta-analysis, pre-existing CHF was observed to modify the association between $\mathrm{SCH}$ and $\mathrm{CHF}$ events. Given the impact of low serum thyrotropin level on cardiac contractility, systemic vascular resistance, electrophysiologic irritability, and atherosclerosis, it has been speculated that subclinical hypothyroidism might predispose to death from cardiac causes. It is plausible that subpopulations with CHF may be predisposed to the cardiac morbidity and mortality associated with subclinical hypothyroidism, owing to underlying distortions in ventricular architecture, alterations in neurohormonal activation, and vascular tone [6].

To better understand the SCH role in CCF patients, this is a study to evaluate the impact of SCH on CCF patients during hospitalization. As there is a rising concern of $\mathrm{SCH}$, we also determined the hospital characteristics that had a higher odds of the codiagnosis of SCH in CCF patients.

\section{Materials And Methods}

\section{Data source}

In the study mentioned below, a retrospective analysis was performed using the healthcare cost and utilization project (HCUP) nationwide inpatient sample (NIS) data [7]. The agency for healthcare research and quality (AHRQ) sponsors the HCUP databases that are specifically designed to determine and identify patterns in utilization and cost across the United States hospitals. The HCUP-NIS database is the largest inpatient database available in the United States, which represents a sample of non-federal United States community hospitals. The sample size available via the database further facilitates the recognition and analyses of rare conditions and special patient populations. The patients, physicians, hospitals, state, and hospital identifiers are de-identified, to protect the privacy of the individual. Also, there are many clinical and non-clinical hospitalization data elements recorded in the HCUP NIS database. The sample of nonclinical information includes the patient's demographic data, hospital characteristics, and total inpatient charges. For example, the clinical-related information includes primary, and other, diagnoses, disposition status, and length of inpatient stay.

\section{Variables of interest}

Based on the International Classification of Diseases, 9th Edition, Clinical Modifications (ICD-9-CM) diagnosis codes, we identified the controls with a primary diagnosis of CCF at the time of admission. Correspondingly, based on the ICD-9-CM diagnosis codes, patients with a primary diagnosis of CCF and a secondary diagnosis of SCH at the time of admission were identified as the cases. In HCUP databases, more than 14,000 ICD-9-CM diagnosis codes had been mentioned. CCF, non-hypertensive type, was identified using diagnosis code 398.91, 428.0, 428.1, 428.20-428.23, 428.30-428.33, 428.40-428.43, or 428.9. Both iatrogenic hypothyroidism and acquired hypothyroidism are part of SCH [3], and, so, SCH was identified using ICD-9-CM diagnosis codes 244.3 (iatrogenic hypothyroidism) and 244.8 (other, specified, acquired hypothyroidism).

To measure the differences in hospitalization outcomes in CCF patients versus CCF with SCH patients, the outcome variables of interest included the severity of illness, which measures the loss of body functions, inpatient length of stay, inpatient total charges, disposition of the patient, and in-hospital mortality. In the NIS, we defined death as in-hospital mortality and, in this paper, it is reported as all-cause. We calculated 
the inpatient length of stay as the number of nights the patient remained in the hospital for a particular discharge and the inpatient stay was all-cause in this analysis. Inpatient total charges during hospitalization do not include professional fees and non-covered charges. If the source provided total inpatient charges with professional fees, the professional fees were removed from the charge during HCUP processing.

\section{Case and control subject selection}

Cases were selected from among all discharges in the NIS from 2012-2014 with a primary diagnosis of CCF, the secondary diagnosis of SCH and age > 18 years. Controls were selected from among all discharges in the NIS from 2012-2014 with a primary diagnosis of CCF and were matched with the cases for age, gender, and race.

\section{Statistical analysis}

In this study, exploratory data analysis was performed using cross-tabulation over the NIS database focusing on the patient with CCF and SCH and the use of descriptive statistics to summarize the results. Pearson's chi-square test and independent sample T-test were used for categorical data and continuous data, respectively. Multilevel logistic regression with random effects for hospital variability was used to calculate adjusted Odds Ratio (aOR). We used the NIS database to obtain nationally representative inpatient data, and age, gender, and race were estimated. All significance tests were two-sided and a p-value $<0.05$ was used as a reference to determine the statistical significance test result. A statistical analysis was conducted using Statistical Package for the Social Sciences (SPSS) version 23 (IBM, Armonk, NY, US) in this study [8]. Our study database does not contain any patient identification and authentication. Thus, we were not required to take Institution Review Board (IRB) permission for this study.

\section{Results}

\section{Sample characteristics}

A total number of 143,735 CCF patients were enrolled in this case-control study from 2012-2014; 73,440 cases had subclinical hypothyroidism (SCH) and 70,295 controls did not have SCH. About half of the total CCF patients were above 80 years of age $(50.3 \%, \mathrm{~N}=72,270)$ with a very low proportion of patients below 60 years $(11.6 \%, N=16,760)$. The majority of the patients in this study were females $(69.4 \%, N=99,775)$ and Caucasians $(78.8 \%, \mathrm{~N}=110850)$. The demographic distribution of the sample population is mentioned in Table 1. 


\section{Cureus}

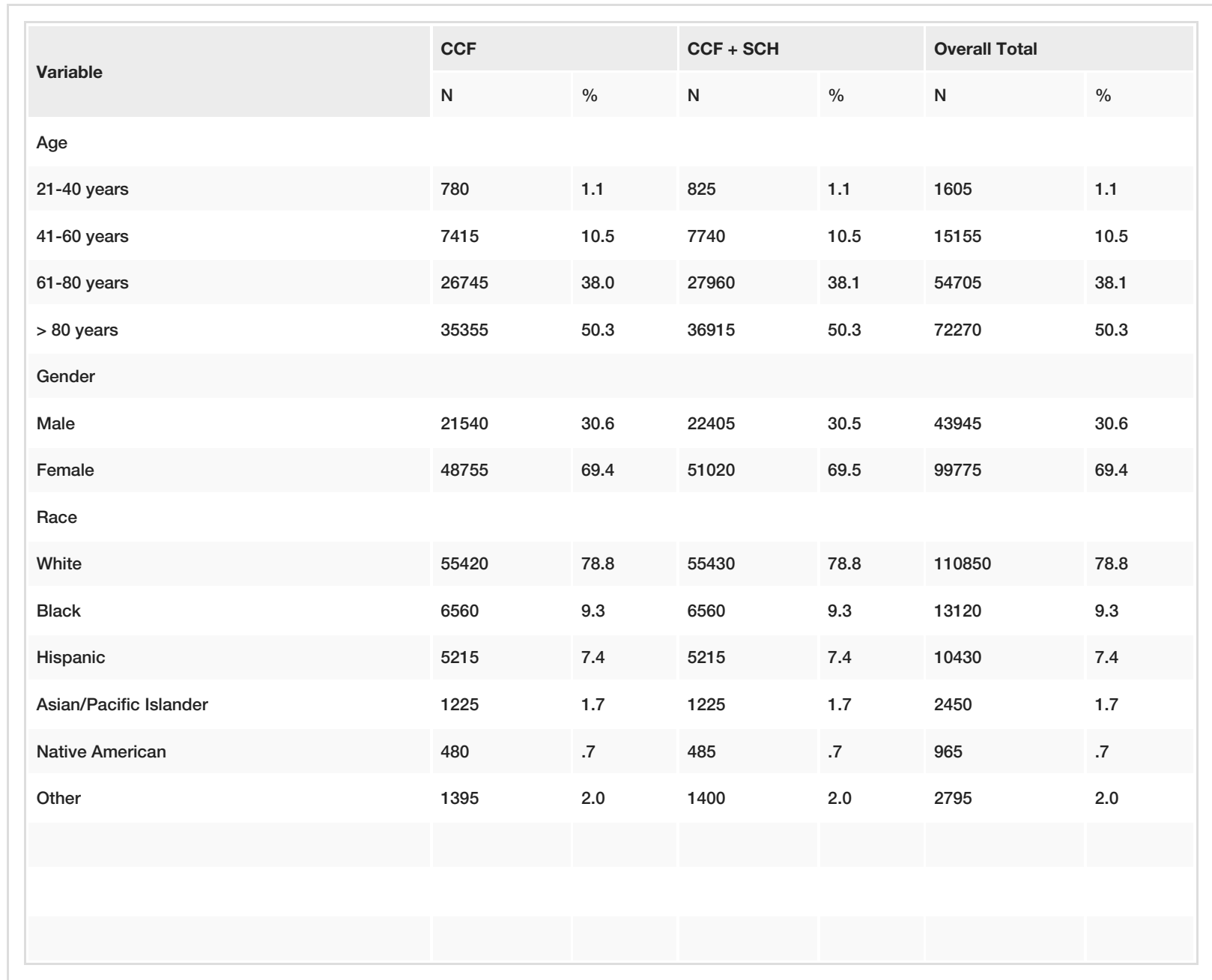

\section{TABLE 1: Demographic distribution in CCF patients by $\mathrm{SCH}$}

CCF: congestive cardiac failure; SCH: subclinical hypothyroidism

\section{Hospitalization outcomes differences per SCH}

About 68,110 CCF patients with SCH (93.1\%) were admitted based on an emergency condition or a nonelective basis, while 5,085 (6.9\%) were admitted on an elective basis. CCF patients with SCH were two times more likely to be admitted on an emergency or elective basis compared to patients without $\mathrm{SCH}(\mathrm{OR}=2.407$, $95 \%$ CI 2.208-2.625, $\mathrm{P}<.001)$.

The median length of inpatient stay per admission for CCF was four days and $31.8 \%$ of SCH patients $(\mathrm{N}=$ 23,370 ) were hospitalized for more than four days compared to 31,435 patients without SCH $(44.7 \%)(\mathrm{P}<$ .001 ). The median hospitalization charges per admission for CCF was $\$ 20,312$. There was not a statistically significant difference between patients with SCH $(49.4 \%, \mathrm{~N}=36,300)$ and without SCH $(49.3 \%, \mathrm{~N}=34,645)$ having hospitalization charges more than $\$ 20,312(\mathrm{P}=.588)$. Thus, CCF patients with SCH had lower odds of longer hospitalization $(\mathrm{OR}=.709,95 \% \mathrm{CI} .660-.762, \mathrm{P}<.001)$ and higher hospitalization charges $(\mathrm{OR}=.783$, $95 \%$ CI .728-.841, P < .001) compared to patients without SCH. The differences in hospitalization outcomes and hospital characteristics are mentioned in Table 2. 


\section{Cureus}

\begin{tabular}{|c|c|c|c|c|c|}
\hline \multirow{2}{*}{ Variable } & \multicolumn{2}{|l|}{ CCF } & \multicolumn{2}{|c|}{$\mathrm{CCF}+\mathrm{SCH}$} & \multirow{2}{*}{$\mathbf{P}$} \\
\hline & $\mathrm{N}$ & $\%$ & $\mathrm{~N}$ & $\%$ & \\
\hline \multicolumn{6}{|l|}{ Admission type } \\
\hline Non-elective & 68430 & 97.4 & 68110 & 93.1 & \multirow{2}{*}{$<.001$} \\
\hline Elective & 1835 & 2.6 & 5085 & 6.9 & \\
\hline \multicolumn{6}{|l|}{ Severity of illness/morbidity } \\
\hline Minor loss of body function & 5470 & 7.8 & 11535 & 15.7 & \multirow{3}{*}{$<.001$} \\
\hline Moderate loss of body function & 28425 & 40.4 & 35855 & 48.8 & \\
\hline Major loss of body function & 36400 & 51.8 & 26050 & 35.5 & \\
\hline \multicolumn{6}{|l|}{ Inpatient stay and cost } \\
\hline Inpatient stay > 4 days (median) & 31435 & 44.7 & 23370 & 31.8 & $<.001$ \\
\hline Inpatient cost > \$20312 (median) & 34645 & 49.3 & 36300 & 49.4 & .588 \\
\hline \multicolumn{6}{|l|}{ In-hospital mortality } \\
\hline Inpatient deaths & 2495 & 3.6 & 1480 & 2.0 & $<.001$ \\
\hline \multicolumn{6}{|l|}{ Bed size of hospital } \\
\hline Small & 19360 & 27.5 & 13470 & 18.3 & \multirow{3}{*}{$<.001$} \\
\hline Medium & 22620 & 32.2 & 18895 & 25.7 & \\
\hline Large & 28315 & 40.3 & 41075 & 55.9 & \\
\hline \multicolumn{6}{|l|}{ Location/teachin status of Hospital } \\
\hline Rural, non-teaching & 6955 & 9.9 & 12035 & 16.4 & \multirow{3}{*}{$<.001$} \\
\hline Urban, non-teaching & 23600 & 33.6 & 28790 & 39.2 & \\
\hline Urban, teaching & 39740 & 56.5 & 32615 & 44.4 & \\
\hline \multicolumn{6}{|l|}{ Region of hospital } \\
\hline Northeast & 69380 & 98.7 & 9395 & 12.8 & \multirow{4}{*}{$<.001$} \\
\hline Midwest & 575 & .8 & 28810 & 39.2 & \\
\hline South & 285 & .4 & 19185 & 26.1 & \\
\hline West & 55 & .1 & 16050 & 21.9 & \\
\hline
\end{tabular}

\section{TABLE 2: Hospital outcomes and characteristics distribution in CCF patients by SCH}

Significant $P \leq 0.05$ at $95 \%$ confidence interval

CCF: congestive cardiac failure; $\mathrm{SCH}$ : subclinical hypothyroidism

A lower proportion of CCF patients with SCH $(35.5 \%, \mathrm{~N}=26,050)$ had a major loss of body function or morbidity compared to $51.8 \%(\mathrm{~N}=36,400)$ patients without SCH $(\mathrm{P}<.001)$. CCF patients with SCH had two times higher odds of minor morbidity $(\mathrm{OR}=2.276 ; 95 \% \mathrm{CI} 2.105-2.462 ; \mathrm{P}<.001)$ but lower odds of major morbidity $(\mathrm{OR}=.783 ; 95 \% \mathrm{CI} .728-.841 ; \mathrm{P}<.001)$. Also, 1480 (2\%) patients with SCH died during hospitalization compared to 2495 patients without SCH $(3.6 \%)(\mathrm{P}<.001)$. CCF patients with SCH had lower odds of in-hospital mortality (OR $=.547 ; 95 \% \mathrm{CI} .496-.604 ; \mathrm{P}<.001)$. The association of adverse hospitalization outcomes in CCF patients with SCH are mentioned in Table 3. 


\section{Cureus}

\begin{tabular}{|c|c|c|c|c|}
\hline \multirow{2}{*}{ Variable } & \multirow{2}{*}{ Odds Ratio } & \multicolumn{2}{|c|}{ 95\% Confidence Interval } & \multirow{2}{*}{$\mathbf{P}$} \\
\hline & & Lower Bound & Upper Bound & \\
\hline Moderate Morbidity & 1.380 & 1.284 & 1.484 & $<.001$ \\
\hline Major Morbidity & .783 & .728 & .841 & $<.001$ \\
\hline Inpatient Stay > 4 days & .709 & .660 & .762 & $<.001$ \\
\hline Inpatient Cost > \$20312 & 1.013 & .944 & 1.087 & .715 \\
\hline In-hospital Mortality & .547 & .496 & .604 & $<.001$ \\
\hline
\end{tabular}

\section{TABLE 3: Association of adverse hospital outcomes in CCF with SCH patients}

Significant $\mathrm{P} \leq 0.05$ at $95 \%$ confidence interval, variables were Agency for Healthcare Research and Quality (AHRQ) comorbidity measures

CCF: congestive cardiac failure; SCH: subclinical hypothyroidism

\section{Hospital predictors for SCH co-diagnosis in CCF}

More than half of the CCF patients with SCH $(55.9 \%, \mathrm{~N}=41,075)$ were seen in large-bed-size hospitals and urban teaching hospitals $(44.4 \%, \mathrm{~N}=32,615)$. Yet, CCF patients with SCH had higher odds of being seen in rural non-teaching hospitals $(\mathrm{OR}=1.696 ; 95 \% \mathrm{CI} 1.572-1.831 ; \mathrm{P}<.001)$. The highest proportion of CCF patients with SCH were seen in hospitals located in the midwestern regions of the US $(39.2 \%, \mathrm{~N}=28,810)$, followed by the southern region $(26.1 \%, N=19,185)$, the western region $(21.9 \%, N=16,050)$, and then the northeastern region of the US $(12.8 \%, \mathrm{~N}=9,395)$. When compared with patients without SCH, CCF patients with SCH had the highest likelihood of presence in the western region of the US ( $\mathrm{OR}=149.924 ; 95 \% \mathrm{CI}$ 110.497-203.419; $\mathrm{P}<.001)$ followed by the southern region ( $\mathrm{OR}=31.431 ; 95 \%$ CI 26.066-37.900; $\mathrm{P}<.001)$. Hospital predictors of SCH in CCF patients are mentioned in Table 4. 


\section{Cureus}

\begin{tabular}{|c|c|c|c|c|}
\hline \multirow{2}{*}{ Variable } & \multirow{2}{*}{ Odds Ratio } & \multicolumn{2}{|c|}{ 95\% Confidence Interval } & \multirow{2}{*}{$\mathbf{P}$} \\
\hline & & Lower Bound & Upper Bound & \\
\hline \multicolumn{5}{|l|}{ Bed size of hospital } \\
\hline Small & .574 & .533 & .619 & $<.001$ \\
\hline Medium & .724 & .673 & .778 & $<.001$ \\
\hline Large & 1.258 & 1.171 & 1.351 & $<.001$ \\
\hline \multicolumn{5}{|c|}{ Location/teaching type of hospital } \\
\hline Rural, non-teaching & 1.696 & 1.572 & 1.831 & $<.001$ \\
\hline Urban, non-teaching & 1.270 & 1.181 & 1.365 & $<.001$ \\
\hline Urban, teaching & .831 & .774 & .892 & $<.001$ \\
\hline \multicolumn{5}{|l|}{ Region of hospital } \\
\hline Northeast & .056 & .048 & .065 & $<.001$ \\
\hline Midwest & 20.340 & 17,223 & 24.022 & $<.001$ \\
\hline South & 31.431 & 26.066 & 37.900 & $<.001$ \\
\hline West & 149.924 & 110.497 & 203.419 & $<.001$ \\
\hline
\end{tabular}

\section{TABLE 4: Hospital predictors for SCH in CCF patients}

Significant $\mathrm{P} \leq 0.05$ at $95 \%$ confidence interval, variables were Agency for Healthcare Research and Quality (AHRQ) comorbidity measures CCF: congestive cardiac failure; SCH: subclinical hypothyroidism

\section{Discussion}

This analysis of population-based hospital data from patients admitted with CCF and CCF with SCH discloses the impact of SCH on hospitalization and related outcomes in CCF. More than half the patients were above the age of 80 years, which may be due to the increase in serum T4 and TSH levels, decreased triiodothyronine (T3) levels, and reverse triiodothyronine (rT3) levels with increasing age. In an earlier study on the thyroid hormone, it has been shown that with aging, the measurement of serum deiodinase levels in a range of healthy adults has demonstrated a significant inverse correlation of 3', 3'-diiodothyronine, 3'.5'diiodothyronine, and 3,5-diiodothyronine levels. Also, it can be seen in elderly individuals due to the increasing incidence and prevalence of autoimmune thyroiditis that occurs with aging [9]. Studies also revealed more SCH in the female population according to the Whickham survey [10]. The prevalence of SCH was three times higher in women than in men. One possible reason for this difference is that the majority of cases of thyroid dysfunction are due to an autoimmune disease, which is more common in women. Therefore, it is necessary to routinely evaluate thyroid function among women during health examinations [10]. According to the American College of Physicians, treatment for subclinical thyroid dysfunction is controversial but suggests that screening to detect thyroid dysfunction may be indicated in women older than 50 years [11].

In an earlier study, the association between CCF patients with SCH shows that a TSH level of $7.0 \mathrm{mIU} / \mathrm{L}$ or greater was predictive of an incident and recurrent CCF events in those without and with a common diagnosis of CHF [12]. The controversy of the clinical benefit of levothyroxine in older patients in an SCH trial on the incidence of cardiovascular events or mortality indicated that treatment with levothyroxine in older persons with subclinical hypothyroidism provided no significant benefits [13].

In our study, a majority of the patients were admitted under emergency conditions and a higher proportion of CCF patients without SCH had a longer inpatient stay compared to patients with $\mathrm{SCH}$, but equal proportions of SCH and non-SCH patients had hospitalization charges higher than the median. A CCF patient with SCH had higher odds of moderate morbidity compared to a patient without SCH. Yet, inhospital mortality was also lower in CCF patients with SCH (2\%) compared to patients without SCH (3.6\%). Moreover, according to the other studies, it demonstrates that patients with SCH were associated with an increased risk for all-cause and cardiovascular mortality in adults [14]. Previous results showed that SCH may increase the risks of hypercholesterolemia and atherosclerosis, and a mildly altered thyroid status has been reported to be associated with an increased risk of mortality in patients with cardiac disease. This 
reported an increased risk of all-cause mortality in SCH patients with comorbid conditions [15]. There is a lot of debate over mortality among CCF patients with $\mathrm{SCH}$, and our study did not appreciate any increase in the association with in-hospital mortality as CCF patients with SCH had lower odds of in-hospital mortality compared to patients without SCH.

A higher proportion of CCF patients with SCH were seen in large-size teaching hospitals in the urban region and the majority of these cases were present in the mid-western region (39.2\%) of the United States. This study also found that CCF patients admitted in rural and non-teaching hospitals have higher odds of having $\mathrm{SCH}$, and these patients in the western region of the United States have a 200 times higher likelihood of a codiagnosis of $\mathrm{SCH}$.

\section{Limitations}

NIS is an organizational database and the data used in these studies lacks the patient-level data needed to form specific clinical associations. Additionally, these types of retrospective case-control studies are always subject to a selection bias, which might be accentuated by the moderate sensitivity of diagnostic codes for CCF and SCH. In this study, we too could not account for the re-hospitalizations of patients, given the nature of the database, although they add to the total inpatient burden. Despite all these restraints, the NIS database provides an excellent population-based perspective on disease associations with systematic and temporal factors and present a rationale for more thorough studies. This study of the dataset is subject to at least a reporting bias, and all information is coded independently of the individual practitioner, making it a probably more reliable source.

\section{Conclusions}

During the hospitalization of patients with CCF and SCH, we observed a variation in hospitalization outcomes, including inpatient length of stay and cost, morbidity, and in-hospital mortality. We found no significant increase in mortality and major morbidity in heart failure patients with subclinical hypothyroidism. There were differences in the hospital characteristics between CCF patients with and without SCH. Thus, hospital bed size, location, and teaching status act as predictors for a co-diagnosis of SCH in CCF. More attention needs to be paid to the elderly population and female sex, which is at a higher risk of SCH. Further research is needed to guide the development of clinical care models for targeting early diagnosis and treatment and to determine whether thyroid hormone replacement would be beneficial for CCF patients with $\mathrm{SCH}$, to both further reduce mortality and morbidity and improve quality of care in these patients.

\section{Additional Information \\ Disclosures}

Human subjects: Consent was obtained by all participants in this study. Animal subjects: All authors have confirmed that this study did not involve animal subjects or tissue. Conflicts of interest: In compliance with the ICMJE uniform disclosure form, all authors declare the following: Payment/services info: All authors have declared that no financial support was received from any organization for the submitted work. Financial relationships: All authors have declared that they have no financial relationships at present or within the previous three years with any organizations that might have an interest in the submitted work. Other relationships: All authors have declared that there are no other relationships or activities that could appear to have influenced the submitted work.

\section{References}

1. Peeters RP: Subclinical hypothyroidism. N Engl J Med. 2017, 376:2556-2565. 10.1056/NEJMcp1611144

2. Fatourechi V: Subclinical hypothyroidism: an update for primary care physicians . Mayo Clin Proc. 2009, 84:65-71.

3. Biondi B, Cooper DS: The clinical significance of subclinical thyroid dysfunction . Endocr Rev. 2008, 29:76131. 10.1210/er.2006-0043

4. Park YJ, Yoon JW, Kim KI, et al.: Subclinical hypothyroidism might increase the risk of transient atrial fibrillation after coronary artery bypass grafting. Ann Thorac Surg. 2009, 87:1846-1852. 10.1016/j.athoracsur.2009.03.032

5. Suh S, Kim DK: Subclinical hypothyroidism and cardiovascular disease . Endocrinol Metab (Seoul). 2015, 30:246-251. 10.3803/EnM.2015.30.3.246

6. Rhee CM, Curhan GC, Alexander EK, Bhan I, Brunelli SM: Subclinical hypothyroidism and survival: the effects of heart failure and race. J Clin Endocrinol Metab. 2013, 98:2326-2336. 10.1210/jc.2013-1039

7. HCUP NIS Database Documentation. Healthcare Cost and Utilization Project (HCUP) . (2018). Accessed: April 15, 2018: https://www.hcup-us.ahrq.gov/nisoverview.jsp.

8. IBM SPSS Statistics for Windows Version 23.0 . (2015). Accessed: April 15, 2018: https://www.ibm.com/products/spss-statistics?lnk=STW_US_MAST_L1_TL\&lnk2=learn_SPSSstatSub.

9. Kim, MI: Hypothyroidism in the Elderly. Endotext [Internet]. De Groot LJ, Chrousos G, Dungan K, et al. (ed): MDText.com, Inc., South Dartmouth, MA; 2000.

10. Tunbridge WM, Evered DC, Hall R, et al.: The spectrum of thyroid disease in a community: the Whickham survey. Clin Endocrinol (Oxf). 1977, 7:481-493. 10.1111/j.1365-2265.1977.tb01340.x 


\section{Cureus}

11. Helfand M, Redfern CC: Clinical guideline, part 2. Screening for thyroid disease: an update. American College of Physicians. Ann Intern Med. 1998, 129:144-158.

12. Rodondi N, Newman AB, Vittinghoff E, de Rekeneire N, Satterfield S, Harris TB, Bauer DC: Subclinical hypothyroidism and the risk of heart failure, other cardiovascular events, and death. Arch Intern Med. 2005, 165:2460-2466. 10.1001/archinte.165.21.2460

13. Stott DJ, Rodondi N, Kearney PM, et al.: Thyroid hormone therapy for older adults with subclinical hypothyroidism. N Engl J Med. 2017, 376:2534-2544. 10.1056/NEJMoa1603825

14. Mitchell JE, Hellkamp AS, Mark DB, et al.: Thyroid function in heart failure and impact on mortality . JACC Heart Fail. 2013, 1:48-55. 10.1016/j.jchf.2012.10.004

15. Tseng FY, Lin WY, Lin CC, Lee LT, Li TC, Sung PK, Huang KC: Subclinical hypothyroidism is associated with increased risk for all-cause and cardiovascular mortality in adults. J Am Coll Cardiol. 2012, 60:730-737. 10.1016/j.jacc.2012.03.047 